nephron

Practice
Nephron 2016;133:111-115

DOI: $10.1159 / 000446666$
Received: February 15, 2016

Accepted after revision: May 5, 2016

Published online: June 7, 2016

\title{
Back to Basics: Is There a Good Reason to Not Systematically Measure Urine Creatinine in Acute Kidney Injury Monitoring?
}

\author{
Alexandre Toledo Maciel on behalf of the Imed Research Group of Investigators \\ Imed Research Group, Intensive Care Unit, Hospital São Camilo Pompéia, São Paulo, Brazil
}

\begin{abstract}
Key Words
Urine creatinine $\cdot$ Creatinine excretion $\cdot$ Creatinine production - Urine output · Acute kidney injury ·

Biochemistry $\cdot$ Monitoring
\end{abstract}

\begin{abstract}
Regardless of the recent advancements in the understanding of the pathophysiology of acute kidney injury (AKI), its diagnosis remains fundamentally dependent on the serum creatinine ( $\mathrm{s} C \mathrm{Cr}$ ) level and urine output (UO), both of which are considered late markers of $\mathrm{AKI}$, offering only a vague idea of the actual creatinine clearance $(\mathrm{CrCl})$. Although not ideal, $\mathrm{CrCl}$ is still the most common alternative of the glomerular filtration rate (GFR) in clinical practice. It is generally accepted that early diagnosis of AKI must reveal kidney impairment before $\mathrm{sCr}$ increases. Much effort has been made to find tubular and glomerular markers of injury which increase (in blood and/or in urine) before the 'official' diagnosis of AKI. Most of these markers are expensive and not widely available, especially in developing countries. Urine creatinine $(\mathrm{CrU})$, the major link between $\mathrm{s} C \mathrm{r}$ and $\mathrm{UO}$, has been systematically ignored and clinicians are usually unaware of its value. The reasons for this are unclear, but it may be related to the lack of a reference range, dependence of its concentration value on the urine flow (which in turn is only adequately assessed with an indwelling urinary catheter) and the clinical unavailability of its counterbalance part - creatinine production. Changes in urine tend to precede changes in blood in the course of AKI development and recovery. Hence, it is
\end{abstract}

important to bear in mind that changes in $\mathrm{s} C \mathrm{r}$ signal renal dysfunction with a significant delay. The search for a more dynamic, 'real-time' but pragmatic assessment of renal function, especially in patients at risk of abrupt decrease in GFR is certainly one of the most relevant focus of research in the field of AKI monitoring. Systematic CrU assessment may be highly relevant in this case.

(c) 2016 S. Karger AG, Basel

\section{Introduction}

Increase in the serum creatinine $(\mathrm{sCr})$ level remains the most available marker of acute kidney injury (AKI) even in developed countries. Despite its wide availability, relatively low cost and pragmatism, it is well established that $\mathrm{sCr}$ is a slow response parameter, and is unable to reveal fast and acute changes in the glomerular filtration rate (GFR). Most recent published reports dedicated to an early AKI diagnosis focused on biomarkers of tubular injury for anticipating loss of renal function, before increases in sCr. In a recent paper by Endre et al. [1], dynamic parameters for the assessment of GFR changes, including sCr kinetics and timed urine collections for the calculation of creatinine excretion, were observed to improve the clinical accuracy for an early diagnosis of delayed graft function (DGF) in recipients of deceased-donor kidney transplant. This postoperative period is a typical scenario for the observation of significant decreases in GFR. Most importantly, the published report highlights some of the

Dr. Alexandre Toledo Macie 


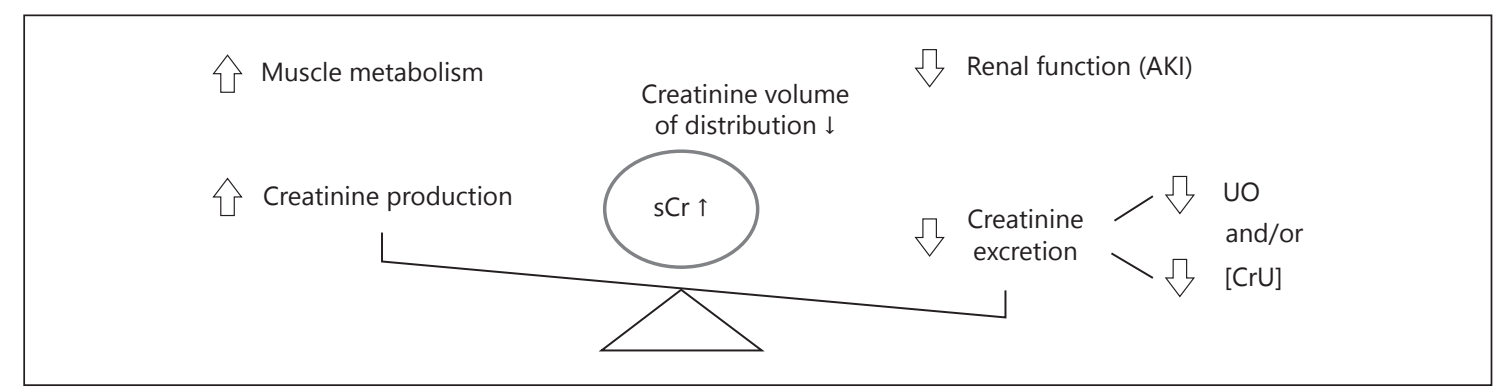

Fig. 1. Multiple variables involved in the determination of serum creatinine ( $\mathrm{sCr}$ ). It is the result of the balance between creatinine production (mainly derived from muscle metabolism, possibly increased in acutely ill patients) and excretion (which is actually the direct marker of renal function). Creatinine excretion in turn depends not only on urine output (UO) but also on the urine creatinine concentration $(\mathrm{CrU})$, usually neglected in clinical practice, generating a gap in real-time renal function evaluation. Since sCr is a concentration value, it also depends on the creatinine volume of distribution (gray circle). In this figure, we demonstrate alterations in the variables that could lead to increases in sCr being its final value always the product of the alterations in all these variables together. AKI = Acute kidney injury.

ple words, creatinine is increasing in blood because it is not being adequately excreted in urine. The problem may be low urine volume, low $\mathrm{CrU}$ concentration or both so that the total mass of excreted creatinine is proportionally low in relation to creatinine production (fig. 1). Using these simple principles, it is easy to understand the 'nonoliguric' $\mathrm{AKI}$ in which low $\mathrm{CrU}$ concentrations are expected and sCr increases even with supposedly normal UO. It also explains conditions of patients with decreased UO but persistently normal sCr levels. Most of these patients have very high concentrations of $\mathrm{CrU}$ and/or very low creatinine production (low muscle mass and low metabolism, for instance, as frequently observed in the elderly).

\section{Which One Is the Real Threat: Oliguria or Low CrU Excretion?}

It is well established that oliguria carries a worse prognosis, independent of $\mathrm{sCr}$ and perhaps may anticipate AKI diagnosis [3]. The lower the UO, the worse is the outcome. However, is the oliguria-associated poor prognosis a matter of urine flow itself or a matter of the capability to excrete creatinine? Since urine is the main way for excreting creatinine, $\mathrm{UO}$ and $\mathrm{sCr}$ must not be evaluated as 2 completely distinct parameters. For the same degree of oliguria and same $\mathrm{sCr}$, it is possible that the outcome may be related at least in part to different $\mathrm{CrU}$ concentrations. Most cases of oliguria without increases in sCr are mild [4] (AKIN stage 1 or RIFLE R) because, with more significant decreases in urine flow, even maximal concentration of $\mathrm{CrU}$ would not be able to prevent decreases in creatinine excretion and increases in $\mathrm{sCr}$, especially in hypercatabolic patients. In oth- 
er words, for a constant creatinine production and volume distribution of creatinine, the $\mathrm{CrU}$ concentration is the variable that, in association with $\mathrm{UO}$, will dictate the subsequent changes in $\mathrm{sCr}$. Also, once $\mathrm{sCr}$ has increased, this statement is no longer true. A gradual increase in creatinine excretion may occur proportionally to the gradual increase in sCr [5, 6]. It is important to emphasize that both $\mathrm{UO}$ and $\mathrm{CrU}$ excretion, although primarily related to GFR, are also influenced to a lesser extent by back-leak, tubuloglomerular feedback, tubular obstruction - mechanisms hardly measured or accessible in clinical practice.

\section{Diuretic Administration Hiding a Decreased Creatinine Excretion}

Diuretic administration usually increases the UO, but should not be considered per se an improvement in kidney function. In cases of AKI fulfilling only the UO criteria, oliguria reversal using diuretics is a potential mask of kidney impairment, possibly jeopardizing $\mathrm{CrCl}$ measurement. Nonetheless, some authors demonstrated no significant diuretic effect on $\mathrm{CrCl}$ determination [7]. Diuretics may alter the total renal blood flow (increasing or decreasing in different situations) and also interfere in intra-renal blood flow distribution, but their use is not expected to increase the creatinine excretion significantly. $\mathrm{CrU}$ measurement is used to determine the real total creatinine excretion and to reveal effective renal function in the presence of diuretics. Increases in UO may be followed by decreases in $\mathrm{CrU}$ concentration, demonstrating no effective improvement in $\mathrm{CrCl}$. This could be the reason why improvement in urine flow per se seems not to improve the renal function or prognosis [8].

The furosemide stress test was proposed for predicting the severity of AKI [9] or the recovery of renal function after continuous renal replacement therapy [10]. Patients with a greater diuretic response are patients with a higher chance of recovery or less risk to progress to a severe stage of AKI. However, these studies have not evaluated the actual creatinine excretion. It would be interesting to know if diuresis is a variable of interest independent of creatinine excretion.

\section{Urine Biochemistry Utility in the Normal sCr Patient}

Since the best approach to early AKI diagnosis is to detect renal impairment while sCr is still normal (or, even better, still not increasing from the baseline), more atten- tion must be given to both urine volume and composition. Biochemical parameters such as urine sodium [11, 12] and the fractional excretion of potassium [13] have been recently proposed to be of help in AKI monitoring, changing before significant increases in sCr. In conjunction with creatinine excretion, systematic assessment of urine electrolytes may signal to some degree of stress to the kidneys and alert to a situation of greater risk of AKI development [14]. In fact, they may suggest a decreasing GFR while $\mathrm{sCr}$ is not yet increasing.

\section{Early AKI Diagnosis: Focusing on the Process Instead of the Result}

It must be emphasized that $\mathrm{UO}$ is a continuous parameter, and $\mathrm{sCr}$ is only measured once or twice a day in most intensive care units (ICUs). Therefore, any variable that is measured continuously has a greater chance of detecting abnormalities at the time they are actually occurring. $\mathrm{UO}$ and $\mathrm{CrU}$ are direct markers of renal function because kidneys produce urine and excrete creatinine. $\mathrm{sCr}$, on the contrary, is an indirect marker of renal function since its increase is a result of renal inability to excrete creatinine properly. Hence, the early detection of renal impairment must first focus on the process (changes in creatinine excretion) and perhaps less on the result of the process (changes in $\mathrm{sCr}$ ). Of course, more frequent $\mathrm{sCr}$ measurement might be an option for early AKI diagnosis, particularly in patients with a high risk of abrupt decreases in GFR [15]. However, this practice has not been proved to improve the prognosis.

In a pragmatic view, $\mathrm{sCr}$ is usually considered more effective than any other measurement of renal function. Even urine flow, the most assessed urinary variable, needs indwelling urine catheter placement and hourly UO measurement. An alternative is UO measurement in blocks of $6 \mathrm{~h}$ [16], which is less time-consuming but not available frequently, especially outside the ICU. On the contrary, $\mathrm{sCr}$ is relatively easy to obtain and interpret although variations in its concentration depend not only on the renal function but also on muscle mass, metabolic rate and creatinine volume of distribution (fig. 1). Physicians must be aware that fluid challenges may decrease sCr merely due to haemodilution [17]. Oscillations in urine volume along the day are often more expected than oscillations in blood volume, which makes the interpretation of $\mathrm{CrU}$ concentration more challenging. Furthermore, there are no well-established reference values for $\mathrm{CrU}$ or for creatinine excretion. Twenty four hours urine collection is 
also inappropriate for measuring abrupt changes in GFR, which may develop in hours. Therefore, the best approach for AKI monitoring in high-risk patients may be to evaluate sequentially (and frequently) not only sCr but also urine flow and $\mathrm{CrU}$, an approach that increases the chance of detecting decreases in $\mathrm{CrCl}$ before any one of these parameters individually. In practical terms, for patients with a urine catheter, a spot urine sample collected at the moment the urine volume is being discarded from the collecting bag would reveal 2 relevant information: the mean urine flow since the last time the bag was emptied $(\mathrm{ml} / \mathrm{h})$ and the mean $\mathrm{CrU}$ concentration in this volume $(\mathrm{mg} / \mathrm{ml}$, for instance). It is then possible to calculate the mean creatinine excretion in $\mathrm{mg} / \mathrm{h}$ during the time that the bag was filling, which must be no longer than a few hours to be effective in the early detection of abrupt decreases in creatinine excretion.

This approach is also valid in AKI recovery monitoring. It is usually detected by decreases in $\mathrm{sCr}$ and/or spontaneous increases in UO. However, analogous to AKI development, decreases in $\mathrm{sCr}$ are the result of the process of AKI recovery, which is actually detected by increases in creatinine excretion. This may occur by increases in $\mathrm{UO}$, but many times this is not enough to reliably predict subsequent decreases in sCr. There are patients who recover urine flow but remain with high levels of sCr. This is usually related to an incapability of the kidney to excrete creatinine properly, and $\mathrm{CrU}$ concentration is usually very low in these patients. There are also cases in which sCr begins to decrease significantly without a significant increase in UO. This is expected to occur when the concentration of $\mathrm{CrU}$ increases consistently, making a similar urine volume more effective in terms of capability to excrete creatinine. An abrupt increase in creatinine excretion is a potential early marker of renal recovery.

\section{CrU Assessment in Daily Practice: Insight Into Early Renal Impairment Recognition in Non-Steady States}

Fast decreases in $\mathrm{CrCl}$ are a major concern, and this is frequent among critically ill patients. $\mathrm{sCr}$ and $\mathrm{UO}$ measurements may overestimate the renal function because they can still be normal while $\mathrm{CrCl}$ is already significantly impaired. Again, one of the major reasons for this to occur is because $\mathrm{CrU}$ is not systematically assessed in combination with sCr and UO. As an example, a $60 \mathrm{~kg}$ patient with sCr of $1.0 \mathrm{mg} / \mathrm{dl}$ and a $U O$ of $60 \mathrm{ml} / \mathrm{h}(1 \mathrm{ml} / \mathrm{kg} / \mathrm{h})$ may have a $\mathrm{CrCl}$ of $100 \mathrm{ml} / \mathrm{min}$ if $\mathrm{CrU}$ is $100 \mathrm{mg} / \mathrm{dl}$ or a $\mathrm{CrCl}$ of 60 $\mathrm{ml} / \mathrm{min}$ if $\mathrm{CrU}$ is $60 \mathrm{mg} / \mathrm{dl}$. It is noteworthy, however, that
$\mathrm{CrCl}$ calculation must be made in a certain period of time (at least a few hours) and it presupposes a stable sCr during this period so that a single value of $\mathrm{sCr}$ is used in the $\mathrm{CrCl}$ formula. In a non-steady state, it is possible that the $\mathrm{sCr}$ increases during this period, making the result of the formula inaccurate. In the recent work of Endre et al. [1], it was proposed that in a non-steady state, $\mathrm{sCr}$ kinetics GFR (KeGFR) and the creatinine excretion to production ratio (E/eG) could help in improving the clinical accuracy for the early diagnosis of DGF in recipients of deceased-donor kidney transplant. They evaluated the occurrence of DGF in the first week post-kidney transplantation measuring KeGFR and E/eG at $4 \mathrm{~h}$ intervals during the first $12 \mathrm{~h}$ after surgery. Both variables enhanced a previously validated clinical model for DGF prediction. Interestingly, E/eG had a better accuracy in predicting DGF than KeGFR.

There are some important differences between KeGFR and $\mathrm{E} / \mathrm{eG}$ : the former is a quantitative parameter and estimates GFR, while the latter is a qualitative parameter, which compares creatinine excretion and production. KeGFR was proposed by Chen [18] as a valuable tool for estimating the kidney function when $\mathrm{sCr}$ is varying acutely. Its formula includes estimated $\mathrm{CrCl}$, creatinine production and volume of distribution using relatively simple and easily assessed variables for facilitating its use at bedside. Its potential role as a GFR alternative was stated but not clearly demonstrated in the paper. Besides the improvement in the (complex) clinical model for DGF prediction, its utility in clinical practice remains highly questionable. Nevertheless, more frequent sCr assessments in situations of expected fast changes in GFR seem advisable [15]. Unfortunately, the absolute values of $\mathrm{sCr}$ at each time point were not mentioned in the study by Endre et al. [1], but the accuracy of their isolated values in predicting DGF was clearly very low.

E/eG formula, originally proposed by Pickering and Mellas [19], also includes the estimated creatinine production, a variable that could be used as a 'benchmark' for the evaluation of creatinine excretion adequacy. However, the determination of creatinine production is probably not solely based on age, sex and weight as stated in the formula by Bjornsson [20]. Fluid challenges are frequently made, particularly in acutely ill patients affecting both their weight and their creatinine volume of distribution. Furthermore, muscle mass and metabolism may vary widely postoperatively and in the presence of critical illness, even considering a stable body weight. It would be difficult to believe in the reliability of the calculated value, which was originally proposed to be used in steady states [20]. Also, creatinine production was considered at a constant rate 
throughout the $12 \mathrm{~h}$ of monitoring. If this is actually true, variations in the $\mathrm{E} / \mathrm{eG}$ value could be mainly attributed to changes in the creatinine excretion rate, which involves both $\mathrm{UO}$ and $\mathrm{CrU}$ concentration during a certain period of time. In other words, abrupt decreases in E/eG would be exclusively the result of acutely reduced creatinine excretion and impaired renal function. A relative decrease is probably more relevant in clinical terms than the absolute values of creatinine excretion. In the study by Endre et al. [1], significantly higher values of E/eG at $12 \mathrm{~h}$ after kidney transplantation in the non-DGF group were probably more a result of higher creatinine excretion than to lower creatinine production. As with $\mathrm{sCr}$, the absolute values of creatinine excretion and production were also not shown, limiting additional analyses.

\section{Conclusion}

$\mathrm{CrU}$ is one of the cornerstones of renal function monitoring, being the (forgotten) link between $\mathrm{sCr}$ and $\mathrm{UO}$. Frequent $\mathrm{CrU}$ concentration assessment, in combination with urine flow and other simple biochemical parameters, have a great potential to improve the applicability of renal physiology in clinical practice. Nonetheless, there are still some limitations to the widespread use of $\mathrm{CrU}$ : lack of reference values, the need for simultaneous evaluation of urine flow (which mandatorily requires a urinary catheter), a potential interference of an increased $\mathrm{sCr}$ in its value and a more precise tool for the assessment of creatinine production, especially in critically ill patients. KeGFR and E/eG proposed by Endre et al. [1], although not that simple to apply at bedside, demonstrate that an approach focused on dynamic changes in $\mathrm{sCr}$ and, more importantly, on dynamic biochemical changes in urine are potential alternatives for the early diagnosis of both AKI development and recovery. Larger studies exploring better the role of urine biochemistry in AKI monitoring are certainly needed.

\section{Disclosure Statement}

The authors declare that they have no conflict of interest.

\section{References}

1 Endre ZH, Pianta TJ, Pickering JW: Timely diagnosis of acute kidney injury using kinetic eGFR and the creatinine excretion to production ratio, E/eG - creatinine can be useful! Nephron 2016;132:312-316.

-2 Mehta RL, Kellum JA, Shah SV, Molitoris BA, Ronco C, Warnock DG, et al: Acute kidney injury network: report of an initiative to improve outcomes in acute kidney injury. Crit Care 2007;11:R31.

- 3 Macedo E, Malhotra R, Bouchard J, Wynn SK, Mehta RL: Oliguria is an early predictor of higher mortality in critically ill patients. Kidney Int 2011;80:760-767.

4 Macedo E: Urine output assessment as a clinical quality measure. Nephron 2015;131:252254.

5 Waikar SS, Sabbisetti VS, Bonventre JV: Normalization of urinary biomarkers to creatinine during changes in glomerular filtration rate. Kidney Int 2010;78:486-494.

6 Ralib AM, Pickering JW, Shaw GM, Devarajan P, Edelstein CL, Bonventre JV, et al: Test characteristics of urinary biomarkers depend on quantitation method in acute kidney injury. J Am Soc Nephrol 2012;23:322-333.

7 Lam NP, Kuk JM, Franson KL, Lau AH: Effect of diuretic drugs on creatinine clearance determination. Ther Drug Monit 1995;17:142-144.
8 Bagshaw SM, Delaney A, Haase M, Ghali WA, Bellomo R: Loop diuretics in the management of acute renal failure: a systematic review and meta-analysis. Crit Care Resusc 2007;9:60-68.

-9 Chawla LS, Davison DL, Brasha-Mitchell E, Koyner JL, Arthur JM, Shaw AD, et al: Development and standardization of a furosemide stress test to predict the severity of acute kidney injury. Crit Care 2013;17:R207.

10 van der Voort PH, Boerma EC, Pickkers P: The furosemide stress test to predict renal function after continuous renal replacement therapy. Crit Care 2014;18:429.

11 Maciel AT, Park M, Macedo E: Physicochemical analysis of blood and urine in the course of acute kidney injury in critically ill patients: a prospective, observational study. BMC Anesthesiol 2013;13:31.

12 Caironi P, Langer T, Taccone P, Bruzzone P, De Chiara S, Vagginelli F, et al: Kidney instant monitoring (K.IN.G): a new analyzer to monitor kidney function. Minerva Anestesiol 2010;76:316-324.

13 Maciel AT, Park M, Macedo E: Fractional excretion of potassium in the course of acute kidney injury in critically ill patients: potential monitoring tool? Rev Bras Ter Intensiva 2014;26:143-147.

14 Maciel AT, Vitorio D: Urine biochemistry assessment in critically ill patients: controversies and future perspectives. J Clin Monit Comput 2016, Epub ahead of print.
15 Maciel AT, Nassar AP Jr, Vitorio DL: Very transient cases of acute kidney injury in the early postoperative period after cardiac surgery: the relevance of more frequent serum creatinine assessment and concomitant urinary biochemistry evaluation. J Cardiothorac Vasc Anesth 2016;30:56-63.

- 16 Macedo E, Malhotra R, Claure-Del Granado R, Fedullo P, Mehta RL: Defining urine output criterion for acute kidney injury in critically ill patients. Nephrol Dial Transplant 2011;26:509-515.

17 Macedo E, Bouchard J, Soroko SH, Chertow GM, Himmelfarb J, Ikizler TA, et al: Fluid accumulation, recognition and staging of acute kidney injury in critically-ill patients. Crit Care 2010;14:R82.

$\checkmark 18$ Chen S: Retooling the creatinine clearance equation to estimate kinetic GFR when the plasma creatinine is changing acutely. J Am Soc Nephrol 2013;24:877-888.

19 Pickering JW, Mellas J: A simple method to detect recovery of glomerular filtration rate following acute kidney injury. Biomed Res Int 2014;2014:542069.

20 Bjornsson TD: Use of serum creatinine concentrations to determine renal function. Clin Pharmacokinet 1979;4:200-222. 